\title{
Comparative metagenomic discovery of the dynamic cellulose-degrading process from a synergistic cellulolytic microbiota
}

\section{Ming Yang}

Dalian Polytechnic University

Jingjing Zhao

Dalian Polytechnic University

\section{Yue Yuan}

Dalian Polytechnic University

\section{Xiaoyi Chen}

Dalian Polytechnic University

Fan Yang ( $\nabla$ yang_fan@dlpu.edu.cn )

Dalian Polytechnic University

\section{Xianzhen Li ( $\nabla$ xianzhen@mail.com )}

Dalian Polytechnic University https://orcid.org/0000-0003-0191-9484

\section{Research}

Keywords: Comparative metagenome, Dynamic cellulose degradation process, Functional genes, Synergistic cellulolytic microbiota

Posted Date: April 17th, 2020

DOl: https://doi.org/10.21203/rs.3.rs-22654/v1

License: (c) (i) This work is licensed under a Creative Commons Attribution 4.0 International License. Read Full License 


\section{Abstract \\ Background}

As a green and sustainable bioenergy source, cellulose is difficult to degrade due to its large molecular weight and high structural complexity. Many cellulolytic microorganisms can secrete a series of enzymes to synergistically catalyze the cellulose degradation with a high efficiency. However, the inability to cultivate most of them, as well as their spatial diversity and temporal variability, limit the clear understanding of the relative area.

\section{Results}

To reveal the dynamic process of cellulose biodegradation, we cultivated a microbiota (FP) with efficient cellulose-degrading ability and compared the different stages of filter paper degradation. Ion chromatography and comparative metagenomic sequencing revealed that the diversity of FP enhanced as the complexity of hydrolysates increased, and the disturbance of FP was greater in early-intermediate than intermediate-final period. Sporocytophaga myxococcoides and Cohnella sp. CIP 111063 dominated the synergistic degradation of cellulose in early-intermediate and intermediate-late stages, respectively. Totally, 432 genes were annotated to cellulolytic pathways, and 363 and 231 unannotated genes were speculated to be related to the degradation of cellulose to cellodextrin/cello-oligosaccharide and cellobiose, respectively. Finally, according to the temporal changes in hydrolysates, community structure and gene abundances, a dynamic cellulose-degrading pathway was designed, which involved key cellulolytic species and enzymes in FP.

\section{Conclusions}

The cellulose-degrading pathway was established based on the dynamic analysis in microbiota FP, suggesting the collaboration and competition between species during cellulose degradation. Our work should provide a new perspective for the subsequent identification of key cellulolytic strains and enzymes and clarification the mechanism of cellulose biodegradation.

\section{Background}

The shortage of fossil energy followed by environmental problems have become a major global issue. Effective utilization of renewable biomass resources is an ineluctable trend for sustainability of development [1]. Cellulose is one of the most abundant renewable biomasses. Therefore, the development of cellulose-derived renewable energy sources is of great significance for recycling agricultural waste and relieving the shortage of fossil fuels and the greenhouse effect. However, the biodegradation of cellulose is difficult due to its large molecular weight and complex structure, which 
greatly limits its industrial applications [2]. Therefore, it is urgent to clarify the molecular mechanism of cellulose biodegradation and explore efficient cellulose enzymatic hydrolysis systems.

The biodegradation of cellulose is accomplished by a series of cellulases. To date, researchers have proposed various hypotheses regarding the action mechanism of cellulases, including the $\mathrm{C}_{1}-\mathrm{C}_{\mathrm{x}}$ theory, the sequential action hypothesis, and the most popular one, the synergism hypothesis [3]. The synergism hypothesis states that the hydrogen bonds of natural cellulose are destroyed by nonhydrolytic protein factors, forming disordered amorphous cellulose. The amorphous cellulose is then hydrolyzed into dextrin and glucose under the action of a series of enzymes. Three main types of enzymes are involved in the synergistic action: endoglucanase, exoglucanase and beta-glucosidase [4]. Previous studies have shown that the synergistic reaction of cellulases greatly improves the hydrolysis efficiency of cellulose. Irwin et al. [5] found that properly proportioned cellulases led to a 15-fold increase in cellulose degradation activity compared to that of any single cellulase. Cellulase mixtures can be secreted naturally by cellulolytic microbiota. Due to the diversity of natural cellulose substrates and habitats, these communities are biologically diverse, which hampers the elucidation of their structure and function [6].

Traditional studies of microbial community diversity usually apply cultivation-based methods, but these strategies are often biased and fail to cultivate many (or more likely, the majority of) naturally occurring species [6]. As a result of the rapid development of next generation sequencing technology in recent years, metagenomic sequencing has become a powerful strategy for exploring the structure of microbial communities and mining functional genes. Through analyzing the total DNA in a sample, this strategy gives further information allowing strain- and gene-level resolutions [7]. At present, metagenomic sequencing has been widely applied for analyzing cellulolytic microbiota, including microbial communities from rumen, animal guts, soil, and compost [8]. The microbial community always changes temporally and spatially at both the species and gene levels [9]. Previous studies have focused chiefly on the spatial distribution of cellulolytic microbial communities from different habitats or exposed to different treatments. However, few studies have comparatively analyzed the changes in the metagenome of the same biota at different cellulose-degrading stages.

In the present study, we cultured a stable soil-derived microbiota capable of efficiently degrading filter paper. The composition of the fermentation supernatant and the composition, structure and functional genes of the microbiota samples at different filter paper degradation stages were analyzed by ion chromatography (IC) and metagenomic shotgun sequencing, respectively. This work will further elucidate the dynamic changes in the composition and structure of the cellulolytic community during the cellulosedegrading process, revealing the core functional strains, enzymes and metabolic pathways involved in cellulose degradation and clarifying its molecular mechanism.

\section{Results And Discussion}

\section{The complexity of hydrolysates increased during the cellulose-degrading process}


The microbiota FP was obtained from woodland soil and cultivated using filter paper as the solo carbon source. Different stages of filter paper degradation were analyzed and compared. When FP was cultured for 3 days (the early stage, FP.3), a light yellow strain lawn appeared in the central areas of the filter paper. Compared to 3 days of cultivation, the strain lawn deepened in color and expanded after 8 days of culture (the intermediate stage, FP.8). As the fermentation proceeded to 15 days (the final stage, FP.15), some areas of the filter paper appeared pasty or even transparent, suggesting that it was further degraded (Fig. 1). However, as fermentation continued, no further degradation of the filter paper was detected (data not shown). The results indicated that FP was able to grow on filter paper and efficiently utilize cellulose as the sole carbon source. This observation was similar to that reported by Dumova et al. [10], who observed a slight degradation area in filter paper after the microbiota sample was cultured for 7 days.

Previous studies suggest that cellulose is first degraded to cellodextrin, which then continues to be hydrolyzed into short-chain cello-oligosaccharides and cellobiose. Cello-oligosaccharides and cellobiose are eventually broken down into glucose [4]. To further analyze the filter paper hydrolysates at different degradation stages, IC analysis was performed. As shown in Fig. 2, a large number of short-chain saccharide molecules with a degree of polymerization (DP) greater than 15 were detected after the microbiota FP was cultivated in filter paper medium for 3 days. After 8 days of continuous degradation, the short-chain molecules were broken down into cello-oligosaccharides and cellobiose with DP values of 2-6. At the final degradation stage (15 days), the cello-oligosaccharides and cellobiose contents decreased, and the end product, glucose, was observed. All the results suggest that the complexity of the hydrolysates increased during the early-intermediate period of cellulose degradation, which was consistent with a previous report by Haruta et al. [11]. They found that the maximum content of various metabolites occurred when cellulose was degraded by microbiota for 2-5 days and then became stable after 7 days of hydrolysis.

\section{Sequencing data}

After quality filtering, $\sim 59.43$ gigabases $(\mathrm{Gb})$ high-quality data were analyzed in the present study (an average of $\sim 6.60 \mathrm{~Gb}$ per sample). These clean reads were assembled using SOAPdenovo, which resulted in 82,261 scaftigs with a minimum length of $500 \mathrm{bp}$, an N50 length of 37.35 kilobases (Kb), and a maximum length of $50.88 \mathrm{~Kb}$. To evaluate the quality of the assembly, we aligned the reads to the scaftigs of each sample and obtained an average alignment rate of $94.78 \%$. This finding indicates that most of the Illumina reads were assembled into scaftigs. In addition, we mixed all unmapped reads to assemble them and obtained a total of 3,473 scaftigs with an average of $\sim 757 \mathrm{bp}$ in length. The average numbers of 24,991 (FP.3), 29,773 (FP.8), and 33,544 (FP.15) open reading frames (ORFs) were predicted by MetaGeneMark and showed an increasing trend as cellulose degradation proceeded, implying that the biodiversity of the FP community might also increase with the degradation process of cellulose. The ORF sequences for all samples were subjected to clustering, and a nonredundant gene catalog was constructed. A total of 44,829 nonredundant ORFs with $\sim 810$ bp average length and $\sim 36.31$ megabases $(\mathrm{Mb})$ total length were obtained. Among them, a total of 27,604 ( $61.58 \%)$ genes had complete ORFs. The statistics of sequence data for the different treatments is given in Table 1. 


\section{Dynamic shifts in the structure and composition of cellulolytic microbiota FP}

Theoretically, complex carbon sources provide a wide range of nutrition for microorganisms. Cellulose degradation produces a variety of metabolites, such as cellodextrin, cello-oligosaccharides, cellobiose, and glucose. Changes in metabolite composition are likely to cause disturbances in the diversity and structure of a microbiota. To assess such disturbances during the cellulose degradation process, Shannon-Wiener and Simpson indices based on species abundance were calculated for FP samples at different degradation stages, and the Wilcox test was employed to identify significant differences in comparisons. As shown in Table 2, both the Shannon-Wiener and Simpson indices revealed that the species diversity of the microbiota FP significantly increased in the intermediate and final stages compared with the early stage $(p<0.05)$. In addition, the increasing rate of species diversity in the earlyintermediate period was significantly faster than that in the intermediate-final period $(p<0.05)$. The 2dimensional nonmetric multidimensional scaling (NMDS) plot showed that the microbiota FP.3 was well separated from FP.8 and FP.15, which were relatively close to each other (Fig. 3a), suggesting that the structure of FP was disturbed in the early-intermediate period but relatively stable in the intermediate-final period, which coincided well with the alpha diversity results. Considering the changing complexity of hydrolysates during the cellulose-degrading process, it could be deduced that the increased species diversity of FP was probably due to the enhanced metabolite complexity.

As shown in Fig. 3b, Bacteroidetes, Firmicutes and Proteobacteria were the main phyla; they accounted for over $96 \%$ of the abundance in FP samples from different cellulose degradation stages, which was consistent with previous studies $[13,14]$. At the genus level, the genera with a total relative abundance greater than $0.5 \%$ included Sporocytophaga (40.82\%), Cohnella (34.85\%), Achromobacter (4.99\%), Herbaspirillum (5.87\%), Paenibacillus (2.00\%), and Microbacterium (0.54\%). At the species level, Sporocytophaga myxococcoide and Cohnella sp. CIP 111063 were the most dominant species, and the relative abundance of the two species reached $40.82 \%$ and $34.27 \%$, respectively.

Previous studies have shown that natural cellulolytic strains degrade cellulose synergistically together with other microorganisms that utilize hydrolysates (such as glucose and cellobiose) as carbon and energy sources $[2,10]$. Furthermore, there might be a "jungle rule" in micro-ecosystems due to competition for carbon sources in the process of cellulose degradation, resulting in the shift of relative abundance among species [15]. As illustrated in Fig. 4, the relative abundance of microbial taxa from FP.3 and FP.8 exhibited significant differences from each other $(p<0.05)$. Here, microbial taxa in FP could be roughly divided into two types: downregulated and upregulated. The downregulated microorganisms were more likely to be involved in the early stage of cellulose degradation (cellulose to cellodextrin). Notably, almost all downregulated taxa belonged to Bacteroidetes, with Sporocytophaga myxococcoide as the representative species. Bacteroidetes are considered to be highly efficient in carbohydrate metabolism, and a few members in this phylum are reported to have cellulose degrading ability [16]. Previous studies have proven that $S$. myxococcoides can glide rapidly on solid surfaces of cellulose substrates and conduct cellulose degradation [17]. Logically, other downregulated genera, such as Flavobacterium and Chitinophaga, are believed to have functions similar to those of S. myxococcoides. On the other hand, 
upregulated taxa might play important roles in catabolizing metabolites such as cello-oligosaccharides, cellobiose and glucose that are produced in the intermediate degradation stage. Most of the upregulated species were derived from Firmicutes and Proteobacteria. Many members of Firmicutes can produce proteases, cellulases, lipases and other extracellular enzymes, which might contribute to cellulose catabolism [18]. Cohnella and Paenibacillus, two dominant genera from Firmicutes, possess the ability to utilize cellulose, hemicellulose, and cellobiose [19]. Cohnella sp. CIP 111063, which was most dominant in the upregulated species, contains a set of genes encoding cellulases. Proteobacteria are rich in the organic layer of forest soil [20]. The two dominant genera from Proteobacteria were Herbaspirillum and Achromobacter. Herbaspirillum is a typical nitrogen-fixing bacterium in soil [21]. Previous studies identified eight gene clusters from this genus that are involved in cellulose biosynthesis and degradation [22]. As members of Achromobacter, Achromobacter xylosoxidans and denitrificans with a low abundance cannot ferment cellobiose and glucose but are positive in oxidase and catalase activity, they probably play a role in removing other gradually accumulated metabolites that are not directly relevant to cellulose degradation [23]. Additionally, these low-abundance bacteria could serve as a species reservoir, together with other low-abundance species, to enhance the environmental adaptability of the microbiota FP.

\section{Core genes in cellulose catabolism}

The gene annotation results for microbiota FP showed that 1956 (4.36\%) genes belong to carbohydrateactive enzymes (CAZymes), including 1074 glycoside hydrolase (GH) coding genes, 522 carbohydratebinding module (CBM) coding genes, 399 glycosyltransferase (GT) coding genes, 144 carbohydrate esterase (CE) coding genes, 50 polysaccharide lyase $(P L)$ coding genes and 19 auxiliary active enzyme (AA) coding genes [24]. Their relative abundances with different treatments were compared using Metastats analysis, and a heat map of the top 35 CAZymes and CBM families was plotted (Fig. 5a). The results revealed that all the proteins only exhibited significant abundance shifts $(p<0.05)$ in the earlyintermediate period of filter paper degradation, which was consistent with the observation of taxa disturbance. Similarly, these annotated protein families could be classified into upregulated and downregulated types. The upregulated families mainly included GH13, GH51, CE7, AA10, GH55 and CE14, of which only GH51 was related to cellulose hydrolysis [25]. A large proportion of the downregulated families were cellulose degradation-related enzymes and functional modules, including GH3, GH5, GH810, GH16, GH26, GH74, CBM2-4, CBM6, CBM9 and CBM32 [24]. As shown in Fig. 5b, 17,543 (39.14\%) genes in the nonredundant gene catalog were annotated to 3472 Kyoto Encyclopedia of Genes and Genomes (KEGG) orthologies (KOs). A total of 76 KOs showed significant differences across FP.3, FP.8 and FP.15 ( $\mathrm{p}<0.05)$, among which four KOs constituted a cellulose degradation pathway: endo-1,4-beta-Dglucanase (Enzyme Commission (EC) 3.2.1.4), beta-D-glucosidase (EC 3.2.1.21), exo-beta-1,4-glucosidase (EC 3.2.1.74) and cellulose 1,4-beta-cellobiosidase (nonreducing end) (EC 3.2.1.91).

However, 7,177 genes were still unannotated, among which a total of 4,031 genes showed high abundance $(\geq 0.01 \%)$ and species annotation information. To further explore the unannotated genes (UGs) potentially related to cellulose catabolism, cluster analysis was performed using the Short Time- 
series Expression Miner (STEM) algorithm. In total, 432 cellulose degradation-related genes were clustered with UGs based on different abundance variation patterns in the early, intermediate and final cellulose degradation stages. Therefore, UGs that have roles similar to those of annotated genes could probably be selected. As shown in Table 3, four typical patterns with significant abundance variation, including pattern 0 , pattern 4, pattern 11 and pattern 14, were selected. Annotated genes clustered to pattern 0 and pattern 4, with downregulated trends, usually encode cellulases, including $\mathrm{GH} 3, \mathrm{GH} 5, \mathrm{GH} 8$, $\mathrm{GH} 9, \mathrm{GH} 10$ and other CAZymes families and are derived from cellulolytic bacteria, such as Sporocytophaga myxococcoides. Therefore, it could be speculated that 363 UGs from pattern 0 and pattern 4 might be related to cellulose and could be named putative cellulolytic gene 1 (PCG1). Most of the genes in pattern 11 and pattern 14, with upregulated trends, were mainly from Cohnella sp. CIP 111063 and Paenibacillus. Nearly all the annotated genes encoded beta-D-glucosidase and exo-beta-1,4glucosidase, suggesting that the clustered 231 UGs, such as FP.3.1_13197 and FP.3.3_15298, might code for enzymes with similar functions. This type of UGs was named putative cellulolytic gene 2 (PCG2).

\section{Predicted cellulolytic catabolism pathway}

Based on the dynamic analysis of the filter paper hydrolysates, the community structure and composition, and the clustered functional genes of microbiota FP, we constructed a complete cellulose catabolism pathway. As shown in Fig. 5c, cellulose was first hydrolyzed into short-chain cellulose, cellodextrin and cellobiose by enzymes including endo-1,4-beta-D-glucanase, cellulose 1,4-betacellobiosidase (nonreducing end) and a small amount of cellulose 1,4-beta-cellobiosidase (reducing end) (EC 3.2.1.176). Second, short-chain cellulose and cellodextrin ( $D P>15)$ continued to be digested into shorter cello-oligosaccharides and cellobiose. This stage required the synergistic action of a variety of enzymes with different functions. Endo-1,4-beta-D-glucanase and 1,4-beta-cellobiosidases continued to participate in this stage, while beta-D-glucosidase and exo-beta-1,4-glucosidase began to hydrolyze shortchain dextran to continuously produce shorter cello-oligosaccharides and cellobiose [27]. Then, cellooligosaccharides and cellobiose were completely hydrolyzed to glucose and glucose 6-phosphate by beta-D-glucosidase, exo-beta-1,4-glucosidase and phosphocellobiase (EC 3.2.1.86). In addition, a small number of cellobiose dehydrogenases (EC 1.1.99.18) and cellobiose phosphotransferases (EC 2.7.1.205) were also involved in cellobiose metabolism, which catalyzed the biochemical reaction of cellobiose to cellobionolactone and glucose 6-phosphate, respectively. Moreover, the PCGs were also located in this pathway. A similar cellulose biodegradation pathway at the molecular level was previously described by Juturu and Wu [4]. However, they provided no information about the dynamically synergistic action at the gene and species levels. Furthermore, two typical strains, Sporocytophaga myxococcoides and Cohnella sp. CIP 111063 dynamically participated in the synergistic degradation process, and had different functional tendencies in the early-intermediate and intermediate-final stages.

\section{Conclusions}

The cellulolytic function of microbiota FP was clarified by the dynamic analysis of hydrolysates and metagenomes at different cellulose-degrading stages. The biodiversity of FP gradually enhanced as the 
complexity of hydrolysates increased. FP-mediated cellulose degradation was accomplished by the collaboration and competition of two types of taxa, represented by Sporocytophaga myxococcoides and Cohnella sp. CIP 111063 respectively. Based on the deduced cellulolytic function of 432 annotated and 594 unannotated genes, a dynamic cellulose-degrading pathway of FP dominated by two core species was predicted. Our work should be valuable for mining key cellulolytic strains and enzymes and formulating cellulose biodegradation mechanisms.

\section{Methods}

\section{Establishment of the cellulose-degrading microbiota}

Lignocellulose-rich soil samples were collected from woodlands on the campus of Dalian Polytechnic University, China ( $\left.38^{\circ} 58^{\prime} 59.2176^{\prime \prime} \mathrm{N}, 121^{\circ} 31^{\prime} 55.1964^{\prime \prime} \mathrm{E}\right)$. These soil samples were dispersed onto a double-layered filter paper placed on a solid medium, then pressed with a flat-ended glass rod to ensure sufficient contact between the soil and filter paper and incubated at $30^{\circ} \mathrm{C}$. Once the degradation of filter paper occurred, each culture was transferred onto a fresh medium with the same components as above. This subculturing process was repeated 10 times, and a microbiota FP with an efficient cellulolytic ability was established. Further sampling was performed in biological triplicate, with three samples transferred onto the respective solid filter paper medium and three transferred into the respective liquid filter paper medium; the incubation for each culture was conducted at $30^{\circ} \mathrm{C}$ under an aerobic condition. The supernatant was collected from each liquid culture after 3,8 and 15 days of shaking incubation for subsequent IC. Each culture on the solid medium was also sampled after 3, 8 and 15 days of static incubation, and the samples were stored at $-20^{\circ} \mathrm{C}$ for subsequent metagenomic analysis. Whatman No. 1 filter paper was used as the only organic component in the solid/liquid media. The inorganic components of the solid/liquid media contained per liter: $0.5 \mathrm{~g}$ each of $\mathrm{KCl}, \mathrm{NaNO}_{3}$, and $\mathrm{MgSO}_{4} 7 \mathrm{H}_{2} \mathrm{O} ; 0.01 \mathrm{~g}$ of FeSO 4 $7 \mathrm{H}_{2} \mathrm{O}$; and $1 \mathrm{~g}$ of $\mathrm{K}_{2} \mathrm{HPO}_{4}, \mathrm{pH}$ 7.0-7.2. To prepare the solid medium, $20 \mathrm{~g}$ per liter agarose was added.

\section{Ion chromatography}

Cellulose hydrolysates were determined by high-performance anion-exchange chromatography (HPAEC) using an ICS-5000 IC system (Dionex, USA) equipped with a pulsed amperometric detector and a CarboPac PA100 column. The samples were analyzed by gradient elution. A $1.0 \mathrm{~mL} / \mathrm{min}$ of mobile phase flow rate with a $25 \mu \mathrm{L}$ of injection volume was adjusted at $30^{\circ} \mathrm{C}$ of column temperature.

\section{DNA preparation and metagenomic sequencing}

Metagenomic DNA was isolated using a PowerSoil Kit (MoBio, USA) following the manufacturer's recommendations [28]. Nanodrop-1000 (Thermo Scientific, USA) and gel electrophoresis were used to determine the DNA quality. High-quality DNA was then stored at $-20^{\circ} \mathrm{C}$ before library construction.

DNA libraries were constructed using an NEBNext® Ultra ${ }^{\mathrm{TM}}$ DNA Library Prep Kit for Illumina (New England Biolabs, USA) under the manufacturer's protocols. First, $1 \mu \mathrm{g}$ of each metagenomic DNA sample was 
fragmented to a size of $\sim 350$ base pairs (bp) using an ultrasonicator (Covaris, USA). Next, the fragmented DNA was end-polished, A-tailed, and ligated with Illumina adaptors for further polymerase chain reaction (PCR) amplification. The PCR products were then purified using the AMPure XP system (Beckman Coulter, USA); libraries were profiled for size distribution using an Agilent 2100 Bioanalyzer (Agilent Biotechnologies, USA) and quantified by quantitative PCR [29]. Finally, the high-quality libraries were sequenced on a Illumina NovaSeq 6000 platform (Novogene, China) using the paired-end $150 \mathrm{bp}$ sequencing strategy.

\section{Bioinformatic analysis}

Raw reads that contained an $>15$ bp of adapter overlap, $>40$ low-quality bases (Q-value $<38$ ) or $10 \%$ " $\mathrm{N"}$ bases were removed using SOAPnuke [30]. The remaining clean reads from each sample were assembled independently into scaftigs without " $\mathrm{N}$ " bases, using SOAPdenovo2 with a k-mer length of 55 bp [31, 32]. The clean reads from each sample were then mapped against the corresponding scaftigs using SOAPaligner [33]. All unmapped reads were mixed and assembled using SOAPdenovo2 with the same parameters. ORFs were predicted from scaftigs longer than 500 bp using MetaGeneMark [34, 35]. To obtain a nonredundant gene catalog, ORFs of more than $100 \mathrm{bp}$ were clustered at $95 \%$ sequence identity using CD-HIT $[34,36]$. SOAPaligner was used to align the clean reads with the nonredundant gene catalog, and only genes supported by at least three mapped reads were designated as unigenes for further analysis [37]. The unigene abundance was calculated for each sample as the number of reads mapped to the unigene and normalized by unigene length [38]. The unigenes were mapped against the NCBI NR database for taxonomic annotation using blastp in DIAMOND [39], with a maximum e-value of 1e-5. MEGAN [40] was applied to determine the taxonomic level of each unigene using the lowest common ancestor-based algorithm. The abundance of each taxonomic group was estimated by summing the abundance of unigenes assigned to the same feature [41]. The KEGG [42] database was used to evaluate the function and metabolic pathways of the unigenes via blastp in DIAMOND, and data visualization was performed using iPath3 [43]. To mine the carbohydrate-active genes, the unigenes were annotated and selected using a hidden Markov model-based web database for automated CAZyme annotation (dbCAN) $[24,44]$.

\section{Statistical analysis}

The alpha diversity was characterized by calculating the Shannon-Wiener and Simpson indices using Mothur [45]. The R package "agricolae" was used to determine significant differences $(p<0.05)$ in alpha diversity indices and their change rates between treatments. The beta-diversity for Bray-Curtis dissimilarities was calculated using the "beta_diversity.py" program in QIIME [46], and visualized by NMDS, which was performed using the "WGCNA", "stat", and "ggplot2" packages in the R software. Bar plots and heat maps were created using the R packages "ggplot2" and "gplots", respectively. Pairwise comparisons of the taxonomic and functional profiles between treatments were carried out using Metastats [47].

\section{Prediction of gene function and construction of cellulolytic metabolic pathways}


Based on the abundance variation patterns, UGs were clustered together with annotated cellulolytic genes (ACGs) and were thus assigned functions similar to those of ACGs from the same pattern. Furthermore, a dynamic pathway of cellulose degradation was predicted for FP based on the results of IC, annotation and prediction of gene function, and taxonomical annotation. The detailed steps were as follows:

1) UGs and ACGs that displayed no significant difference $(p>0.05)$ in relative abundance between treatments, had a low average relative abundance $(<0.01 \%)$ in all treatments, or were not assigned at the genus level were discarded. Moreover, UGs derived from cellulolytic bacteria were screened out for further analysis.

2) The remaining UGs and ACGs were mixed together and clustered based on the abundance variation patterns using the STEM algorithm [48]. Abundance variation patterns with fewer than 10 ACGs were removed. UGs clustered with major ACGs ( $\geq 60 \%$ ) that had only one certain function, were presumed to have the same function.

3) To establish the cellulolytic pathway for FP, the degradation products that were determined by IC were first mapped to the cellulolytic pathway according to the order of degradation. Subsequently, in combination with the pathways and reactions described in the KEGG database, ACGs and PCGs were mapped to the cellulolytic pathway of FP based on the annotated and putative functions, respectively.

\section{Abbreviations}

FP: Filter paper-degrading microbiota; IC: Ion chromatography; FP.3, FP.8 and FP.15: Microbiota FP cultivated in filter paper medium for 3, 8, and 15 days; DP: Degree of polymerization; Gb: Gigabases; Kb: Kilobases; ORF: Open reading frame; Mb: Megabases; NMDS: Nonmetric multidimensional scaling; CAZyme: Carbohydrate-active enzyme, GH: Glycoside hydrolase; CBM: Carbohydrate-binding module; GT: Glycosyltransferase; CE: Carbohydrate esterase; PL: Polysaccharide lyase; AA: Auxiliary activities; KEGG: Kyoto Encyclopedia of Genes and Genomes; KO: KEGG orthology; EC: Enzyme commission number; UG: Unannotated gene; STEM: Short Time-series Expression Miner; PCG: Putative cellulolytic gene; HPAEC: High-performance anion-exchange chromatography; PCR: Polymerase chain reaction; dbCAN: Database for automated carbohydrate active enzyme annotation; ACG: Annotated cellulolytic gene.

\section{Declarations}

\section{Acknowledgements}

Not applicable.

\section{Author's contributions}

MY: designed the strategy for metagenomic analysis, developed the scripts and wrote the manuscript. JZ: contributed with reagents, materials, and analyses, performed the experiments, and wrote the manuscript. 
YY, XC: contributed with reagents, materials, and performed the experiments. FY, XL: drafted, wrote and revised the manuscript. All authors agreed with the submitted version of the paper. All authors read and approved the final manuscript.

\section{Funding}

This work was supported by the Natural Sciences Foundation of China (31671796, 31771907, and 31801469), and the Liaoning BaiQianWan Talents Program is also gratefully acknowledged.

\section{Availability of data and materials}

The metagenomic reads generated in this study have been submitted to NCBI Sequence Read Archive (SRA) under the accession number SRP255666.

\section{Ethics approval and consent to participate}

Not applicable.

\section{Consent for publication}

Not applicable.

\section{Competing interests}

The authors declare that they have no competing interests.

\section{Author details}

School of Biological Engineering, Dalian Polytechnic University, Ganjingziqu, Dalian 116034, PR China.

\section{References}

1. Rodionova MV, Poudyal RS, Tiwari I, Voloshin RA, Zharmukhamedov SK, Nam HG, et al. Biofuel production: challenges and opportunities. Int J Hydrogen Energ. 2017;42:8450-61.

2. Jayasekara S, Ratnayake R. Microbial Cellulases: An Overview and Applications. In: Rodriguez A, Eugenio ME, editors. Cellulose. London: IntechOpen; 2019. p. 84531.

3. Zhang YH, Lynd LR. Toward an aggregated understanding of enzymatic hydrolysis of cellulose: noncomplexed cellulase systems. Biotechnol Bioeng. 2004;88:797-824.

4. Juturu V, Wu JC. Microbial cellulases: Engineering, production and applications. Renew Sust Energ Rev. 2014;33:188-203.

5. Irwin DC, Spezio M, Walker LP, Wilson DB. Activity studies of eight purified cellulases: specificity, synergism, and binding domain effects. Biotechnol Bioeng. 1993;42:1002-13.

6. Wilson DB. Microbial diversity of cellulose hydrolysis. Curr Opin Microbiol. 2011;14:259-63. 
7. Knight R, Vrbanac A, Taylor BC, Aksenov A, Callewaert C, Debelius J, et al. Best practices for analysing microbiomes. Nat Rev Microbiol. 2018;16:410-22.

8. Tiwari R, Nain L, Labrou NE, Shukla P. Bioprospecting of functional cellulases from metagenome for second generation biofuel production: a review. Crit Rev Microbiol. 2018;44:244-57.

9. Mande SS, Mohammed MH, Ghosh TS. Classification of metagenomic sequences: methods and challenges. Brief Bioinform. 2012;13:669-81.

10. Dumova VA, Kruglov YV. A cellulose-decomposing bacterial association. Microbiology. 2009;78:2349.

11. Haruta S, Cui Z, Huang Z, Li M, Ishii M, Igarashi Y. Construction of a stable microbial community with high cellulose-degradation ability. Appl Microbiol Biotechnol. 2002;59:529-34.

12. Miller JR, Koren S, Sutton G. Assembly algorithms for next-generation sequencing data. Genomics. 2010;95:315-27.

13. Berlemont R, Allison SD, Weihe C, Lu Y, Brodie EL, Martiny JB, et al. Cellulolytic potential under environmental changes in microbial communities from grassland litter. Front Microbiol. 2014;5:639.

14. Rosnow JJ, Anderson LN, Nair RN, Baker ES, Wright AT. Profiling microbial lignocellulose degradation and utilization by emergent omics technologies. Crit Rev Biotechnol. 2016;37:626-40.

15. Hibbing ME, Fuqua C, Parsek MR, Peterson SB. Bacterial competition: surviving and thriving in the microbial jungle. Nat Rev Microbiol. 2010;8:15-25.

16. Taillefer M, Arntzen MO, Henrissat B, Pope PB, Larsbrink J. Proteomic Dissection of the Cellulolytic Machineries Used by Soil-Dwelling Bacteroidetes. mSystems. 2018;3:e00240-18.

17. Berg B, von Hofsten B, Pettersson G. Electronmicroscopie observations on the degradation of cellulose fibres by Cellvibrio fulvus and Sporocytophaga myxococcoides. J Appl Bacteriol. 1972;35:215-9.

18. Lim JW, Chiam JA, Wang JY. Microbial community structure reveals how microaeration improves fermentation during anaerobic co-digestion of brown water and food waste. Bioresour Technol. 2014;171:132-8.

19. Fathallh Eida M, Nagaoka T, Wasaki J, Kouno K. Isolation and Characterization of Cellulosedecomposing Bacteria Inhabiting Sawdust and Coffee Residue Composts. Microbes Environ. 2012;27:226-33.

20. Llado S, Lopez-Mondejar R, Baldrian P. Forest soil bacteria: diversity, involvement in ecosystem processes, and response to global change. Microbiol Mol Biol Rev. 2017;81:e00063-16.

21. Schmid M, Baldani JI, Hartmann A. The Genus Herbaspirillum. In: Dworkin M, Falkow S, Rosenberg E, Schleifer K-H, Stackebrandt E, editors. The Prokaryotes. New York: Springer; 2006. pp. 141-50.

22. Monteiro RA, Balsanelli E, Tuleski T, Faoro H, Cruz LM, Wassem R, et al. Genomic comparison of the endophyte Herbaspirillum seropedicae SmR1 and the phytopathogen Herbaspirillum rubrisubalbicans $\mathrm{M} 1$ by suppressive subtractive hybridization and partial genome sequencing. FEMS Microbiol Ecol. 2012;80:441-51. 
23. Weitkamp JH, Tang YW, Haas DW, Midha NK, Crowe JE Jr. Recurrent Achromobacter xylosoxidans bacteremia associated with persistent lymph node infection in a patient with hyper-immunoglobulin M syndrome. Clin Infect Dis. 2000;31:1183-7.

24. Lombard V, Ramulu HG, Drula E, Coutinho PM, Henrissat B. The Carbohydrate-active enzymes database (CAZy) in 2013. Nucl Acids Res. 2014;42:D490-5.

25. Sukharnikov LO, Cantwell BJ, Podar M, Zhulin IB. Cellulases: ambiguous nonhomologous enzymes in a genomic perspective. Trends Biotechnol. 2011;29:473-9.

26. Nedashkovskaya OI, Kim SB. Pontibacter. Bergey's Manual of Systematics of Archaea and Bacteria. Hoboken: John Wiley \& Sons, Inc.; 2015. pp. 1-4.

27. Mba Medie F, Davies GJ, Drancourt M, Henrissat B. Genome analyses highlight the different biological roles of cellulases. Nat Rev Microbiol. 2012;10:227-34.

28. Mohiuddin MM, Salama Y, Schellhorn HE, Golding GB. Shotgun metagenomic sequencing reveals freshwater beach sands as reservoir of bacterial pathogens. Water Res. 2017;115:360-9.

29. Wang JH, Lu J, Zhang YX, Wu J, Luo Y, Liu H. Metagenomic analysis of antibiotic resistance genes in coastal industrial mariculture systems. Bioresour Technol. 2018;253:235-43.

30. Chen Y, Chen Y, Shi C, Huang Z, Zhang Y, Li S, et al. SOAPnuke: a MapReduce acceleration-supported software for integrated quality control and preprocessing of high-throughput sequencing data. Gigascience. 2018;7:1-6.

31. Luo R, Liu B, Xie Y, Li Z, Huang W, Yuan J, et al. SOAPdenovo2: an empirically improved memoryefficient short-readde novoassembler. Gigascience. 2012;1:18.

32. Nan Q, Fengling Y, Ang L, Edi P, Yanfei C, Li S, et al. Alterations of the human gut microbiome in liver cirrhosis. Nature. 2014;513:59-64.

33. Li R, Yu C, Li Y, Lam TW, Yiu SM, Kristiansen K, et al. SOAP2: an improved ultrafast tool for short read alignment. Bioinformatics. 2009;25:1966-7.

34. Sunagawa S, Coelho LP, Chaffron S, Kultima JR, Labadie K, Salazar G, et al. Structure and function of the global ocean microbiome. Science. 2015;348:1261359.

35. Zhu W, Lomsadze A, Borodovsky M. Ab initio gene identification in metagenomic sequences. Nucl Acids Res. 2010;38:e132.

36. Li W, Godzik A. Cd-hit: a fast program for clustering and comparing large sets of protein or nucleotide sequences. Bioinformatics. 2006;22:1658-9.

37. Qin J, Li R, Raes J, Arumugam M, Burgdorf KS, Manichanh C, et al. A human gut microbial gene catalogue established by metagenomic sequencing. Nature. 2010;464:59-65.

38. Villar E, Farrant GK, Follows M, Garczarek L, Speich S, Audic S, et al. Environmental characteristics of Agulhas rings affect interocean plankton transport. Science. 2015;348:1261447.

39. Buchfink B, Xie C, Huson DH. Fast and sensitive protein alignment using DIAMOND. Nat Methods. 2015;12:59-60. 
40. Huson DH, Mitra S, Ruscheweyh HJ, Weber N, Schuster SC. Integrative analysis of environmental sequences using MEGAN4. Genome Res. 2011;21:1552-60.

41. Li J, Jia H, Cai X, Zhong H, Feng Q, Sunagawa S, et al. An integrated catalog of reference genes in the human gut microbiome. Nat Biotechnol. 2014;32:834-41.

42. Kanehisa M, Araki M, Goto S, Hattori M, Hirakawa M, Itoh M, et al. KEGG for linking genomes to life and the environment. Nucleic Acids Res. 2008;36:D480-4.

43. Darzi Y, Letunic I, Bork P, Yamada T. iPath3.0: interactive pathways explorer v3. Nucl Acids Res. 2018;46:W510-3.

44. Yin Y, Mao X, Yang J, Chen X, Mao F, Xu Y. dbCAN: a web resource for automated carbohydrate-active enzyme annotation. Nucl Acids Res. 2012;40:W445-51.

45. Schloss PD, Westcott SL, Ryabin T, Hall JR, Hartmann M, Hollister EB, et al. Introducing mothur: Open-Source, Platform-Independent, Community-Supported Software for Describing and Comparing Microbial Communities. Appl Environ Microbiol. 2009;75:7537-41.

46. Caporaso JG, Kuczynski J, Stombaugh J, Bittinger K, Bushman FD, Costello EK, et al. QIIME allows analysis of high-throughput community sequencing data. Nat Methods. 2010;7:335-6.

47. White JR, Nagarajan N, Pop M. Statistical methods for detecting differentially abundant features in clinical metagenomic samples. PLoS Comput Biol. 2009;5:e1000352.

48. Ernst J, Bar-Joseph Z. STEM: a tool for the analysis of short time series gene expression data. BMC Bioinformatics. 2006;7:191.

\section{Tables}

Table 1 Sequencing, assembly and annotation statistics.

\begin{tabular}{|c|c|c|c|}
\hline Metagenome & $\mathrm{FP} .3^{\mathrm{a}}$ & FP. $8^{\mathrm{a}}$ & FP. $15^{\mathrm{a}}$ \\
\hline Sequenced reads (PE150) & $43,883,418 \pm 1,855,880$ & $45,679,087 \pm 1,501,271$ & $42,587,865 \pm 1,709,086$ \\
\hline Reads after quality filtering (PE150) & $43,858,031 \pm 1,850,587$ & $45,658,274 \pm 1,503,574$ & $42,554,324 \pm 1,705,403$ \\
\hline Scaftigs $\geq 500 \mathrm{bp}$ & $6,708 \pm 283$ & $10,467 \pm 1,368$ & $10,245 \pm 962$ \\
\hline Reads used & $41,570,112 \pm 1,753,145$ & $43,296,086 \pm 1,425,135$ & $40,334,498 \pm 1,615,819$ \\
\hline $\mathrm{N} 50^{\mathrm{b}}$ scaftig size (bp) & $30,059 \pm 5,114$ & $7,977 \pm 1,485$ & $9,213 \pm 93$ \\
\hline Predicted non-redundant $\mathrm{ORFs}^{\mathrm{c}}$ & $24,991 \pm 322$ & $29,773 \pm 1,004$ & $33,544 \pm 1,705$ \\
\hline Total ORF Length (Mb) & $19.765 \pm 0.469$ & $22.155 \pm 0.029$ & $27.160 \pm 0.776$ \\
\hline Average ORF length (bp) & $798 \pm 10$ & $744 \pm 24$ & $783 \pm 9$ \\
\hline Assigned putative function ${ }^{d}$ & $24,342 \pm 305$ & $28,739 \pm 977$ & $32,335 \pm 1,602$ \\
\hline Assigned carbohydrate-active enzymes ${ }^{\mathrm{e}}$ & $967 \pm 25$ & $992 \pm 80$ & $1246 \pm 97$ \\
\hline
\end{tabular}

a FP.3, FP.8 and FP.15 refer to FP microbiota cultivated in filter paper medium for 3, 8, and 15 days, respectively.

${ }^{\mathrm{b}} \mathrm{N} 50$ is the minimum scaftigs length for the first 50\% of the assembly after sorting in descending order of their lengths [12]. 
${ }^{\mathrm{c}}$ ORF: open reading frame.

${ }^{\mathrm{d}}$ Assigned putative functions is the number of ORF annotated to putative functions through searching in NCBI NR database.

e Assigned carbohydrate-active enzymes is the number of ORF assigned to carbohydrate-active enzymes (CAZy) database.

Values represent means \pm standard error.

Table 2 Alpha diversity indices of different FP microbiota samples.

\begin{tabular}{lll}
\hline Alpha Diversity* & Shannon-Wiener & Simpson \\
\hline FP.3 & $1.540 \pm 0.023 \mathrm{~b}$ & $0.367 \pm 0.012 \mathrm{a}$ \\
FP.8 & $1.695 \pm 0.012 \mathrm{a}$ & $0.305 \pm 0.003 \mathrm{~b}$ \\
\hline FP.15 & $1.766 \pm 0.008 \mathrm{a}$ & $0.288 \pm 0.002 \mathrm{~b}$ \\
\hline Normalized Differential Alpha Diversity† & Shannon-Wiener (day- 1$)$ & Simpson (day-1) \\
\hline FP.3 vs FP.8 & $0.0311 \pm 0.0047 \mathrm{a}$ & $-0.01227 \pm 0.00250 \mathrm{a}$ \\
FP.8 vs FP.15 & $0.0102 \pm 0.0014 \mathrm{~b}$ & $-0.00248 \pm 0.00048 \mathrm{~b}$ \\
\hline
\end{tabular}

Within a single column, statistical differences of the values between the treatments were labeled with different letters $(\mathrm{P}<0.05)$. Values represent means \pm standard error.

${ }^{*}$ FP.3, FP.8 and FP.15 refer to FP microbiota cultivated in fiber medium for 3, 8, and 15 days, respectively.

${ }^{\dagger}$ Normalized Differential Alpha Diversity is the area difference between the latter and the former alpha diversity indexes divided by the time lag.

Table 3 Top genes belong to 4 different abundance disturbing patterns across treatment FP.3, FP.8, and FP.15. 


\begin{tabular}{llllll}
\hline Abundance & Gene ID & EC Number & CAZy Family & Annotation or putative information & Species \\
disturbing & & & \\
pattern $^{\mathrm{a}}$ & & & \\
\hline
\end{tabular}

Top 10 genes belong to abundance disturbing pattern 0 .

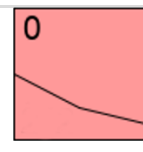

\begin{tabular}{lll} 
FP.8.2_21397 & NA & NA \\
\hline NOVO_MIX_965 & NA & NA \\
& & \\
\hline
\end{tabular}

NA

NA

FP.15.1_36486

NA

FP.15.1_4658 NA

\begin{tabular}{lll} 
FP.15.1_4658 NA & NA \\
\hline FP.15.3_14026 NA & G
\end{tabular}

\begin{tabular}{ll} 
FP.15.3_14026 NA & \\
\hline &
\end{tabular}

FP.15.2_16874 NA

NA

NA

NA

FP.15.1_27227 NA
GH3

CBM4/GH10

GH3

Related to the initial phase of cellulose degradation.

Related to the initial phase of cellulose degradation.

Related to the initial phase of cellulose degradation.

Related to the initial phase of cellulose degradation.

Related to the initial phase of cellulose degradation.

\&beta;-glucosidase (EC 3.2.1.21); glucan

1,4-\&beta;-glucosidase (EC 3.2.1.74)

Binding of these modules has been demonstrated with xylan, \&beta;-1,3glucan, \&beta;-1,3-1,4-glucan, \&beta;-1,6glucan and amorphous cellulose but not with crystalline cellulose; endo-1,4\&beta;-xylanase (EC 3.2.1.8);

FP.15.1_17138 NA CBM6

FP.15.1_16493 3.2.1.4

CBM6

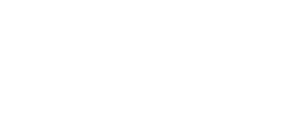

FP.8.1_10150 3.2.1.8/3.2.1.32 CBM6

\section{The}

demonstrated in one case on amorphous cellulose and \&beta;-1,4-xylan. Some of these modules also bind \&beta;-1,3-glucan, \&beta;-1,3-1,4-glucan, and \&beta;-1,4glucan.

The cellulose-binding function has been demonstrated in one case on amorphous cellulose and \&beta;-1,4-xylan. Some of these modules also bind \&beta;-1,3-glucan, \&beta;-1,3-1,4-glucan, and \&beta;-1,4glucan. endoglucanase (EC 3.2.1.4); \&beta;- Sporocytophaga

glucosidase (EC 3.2.1.21); exo-\&beta;-1,4- myxococcoides $^{\text {bc }}$ glucanase / cellodextrinase (EC 3.2.1.74); cellobiohydrolase (EC 3.2.1.91)

Top 10 genes belong to abundance disturbing pattern 4 .

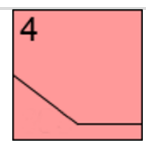

\begin{tabular}{lcc} 
FP.15.1_17147 & NA & NA \\
\hline FP.8.1_9164 & NA & NA \\
\hline FP.15.3_5584 & NA & NA
\end{tabular}

Related to the initial phase of cellulose degradation.

Related to the initial phase of cellulose

degradation.
Related to the initial phase of cellulose
degradation.

[26]

ummariensis $^{\mathrm{b}}$

Pedobacter sp. [20]

Leaf194

Sporocytophaga

[17]

myxococcoides ${ }^{\text {bc }}$

Sporocytophaga

[17]

myxococcoides $^{\mathrm{bc}}$

Sporocytophaga

myxococcoides $^{\mathrm{bc}}$

Sporocytophaga

[17]

myxococcoides $^{\mathrm{bc}}$

Sporocytophaga

myxococcoides ${ }^{\mathrm{bc}}$

Sporocytophaga

[17]

myxococcoides $^{\mathrm{bc}}$

Sporocytophaga

[17]

myxococcoides ${ }^{\text {bc }}$

[17]

Page 16/23 


\begin{tabular}{|c|c|c|c|c|c|}
\hline FP.15.2_11349 & NA & NA & $\begin{array}{l}\text { Related to the initial phase of cellulose } \\
\text { degradation. }\end{array}$ & $\begin{array}{l}\text { Sporocytophaga } \\
\text { myxococcoides }{ }^{\mathrm{bc}}\end{array}$ & [17] \\
\hline FP.8.3_12846 & NA & NA & $\begin{array}{l}\text { Related to the initial phase of cellulose } \\
\text { degradation. }\end{array}$ & $\begin{array}{l}\text { Sporocytophaga } \\
\text { myxococcoides }^{\mathrm{bc}}\end{array}$ & [17] \\
\hline FP.15.2_4723 & NA & CBM6 & $\begin{array}{l}\text { Modules of approx. } 120 \text { residues. The } \\
\text { cellulose-binding function has been } \\
\text { demonstrated in one case on amorphous } \\
\text { cellulose and \&beta;-1,4-xylan. Some of } \\
\text { these modules also bind \&beta;-1,3-glucan, } \\
\text { \&beta;-1,3-1,4-glucan, and \&beta;-1,4- } \\
\text { glucan. }\end{array}$ & $\begin{array}{l}\text { Sporocytophaga } \\
\text { myxococcoides }^{\text {bc }}\end{array}$ & [17] \\
\hline FP.15.1_16917 & 3.2.1.4 & GH9 & $\begin{array}{l}\text { endoglucanase (EC 3.2.1.4); \&beta;- } \\
\text { glucosidase (EC 3.2.1.21); exo-\&beta;-1,4- } \\
\text { glucanase / cellodextrinase (EC 3.2.1.74); } \\
\text { cellobiohydrolase (EC 3.2.1.91); }\end{array}$ & $\begin{array}{l}\text { Sporocytophaga } \\
\text { myxococcoides }^{\mathrm{bc}}\end{array}$ & [17] \\
\hline FP.15.1_32432 & 3.2 .1 .21 & GH3 & $\begin{array}{l}\text { \&beta;-glucosidase (EC 3.2.1.21); glucan } \\
\text { 1,4-\&beta;-glucosidase (EC 3.2.1.74) }\end{array}$ & $\begin{array}{l}\text { Sporocytophaga } \\
\text { myxococcoides }^{\mathrm{bc}}\end{array}$ & [17] \\
\hline FP.15.1_29223 & 3.2.1.4 & GH5 & $\begin{array}{l}\text { endo-\&beta;-1,4-glucanase / cellulase (EC } \\
\text { 3.2.1.4); \&beta;-glucosidase (EC 3.2.1.21); } \\
\text { exo-\&beta;-1,4-glucanase } \\
\text { cellodextrinase (EC 3.2.1.74); cellulose } \\
\text { \&beta;-1,4-cellobiosidase (EC 3.2.1.91) }\end{array}$ & $\begin{array}{l}\text { Sporocytophaga } \\
\text { myxococcoides }^{\text {bc }}\end{array}$ & [17] \\
\hline FP.15.1_20282 & 3.2.1.4 & GH8 & cellulase (EC 3.2.1.4) & $\begin{array}{l}\text { Sporocytophaga } \\
\text { myxococcoides }^{\mathrm{bc}}\end{array}$ & [17] \\
\hline
\end{tabular}

Top 10 genes belong to abundance disturbing pattern 11 .

\begin{tabular}{|c|c|c|c|c|c|}
\hline FP.3.1_13197 & NA & NA & $\begin{array}{l}\text { Related to the mid-phase of cellulose } \\
\text { degradation. }\end{array}$ & $\begin{array}{l}\text { Paenibacillus sp. } \\
\text { MY03 }^{\text {b }}\end{array}$ & {$[2]$} \\
\hline FP.15.1_26209 & NA & NA & $\begin{array}{l}\text { Related to the mid-phase of cellulose } \\
\text { degradation. }\end{array}$ & $\begin{array}{l}\text { Paenibacillus sp. } \\
\text { MY03 }^{\text {b }}\end{array}$ & {$[2]$} \\
\hline FP.3.3_14813 & NA & NA & $\begin{array}{l}\text { Related to the mid-phase of cellulose } \\
\text { degradation. }\end{array}$ & $\begin{array}{l}\text { Cohnella } \\
\text { kolymensis }\end{array}$ & [19] \\
\hline FP.8.3_3197 & NA & NA & $\begin{array}{l}\text { Related to the mid-phase of cellulose } \\
\text { degradation. }\end{array}$ & $\begin{array}{l}\text { Cohnella sp. CIP } \\
111063^{\text {bc }}\end{array}$ & [19] \\
\hline FP.3.3_6193 & NA & NA & $\begin{array}{l}\text { Related to the mid-phase of cellulose } \\
\text { degradation. }\end{array}$ & $\begin{array}{l}\text { Cohnella sp. CIP } \\
111063^{\text {bc }}\end{array}$ & [19] \\
\hline FP.3.3_12658 & 3.2 .1 .21 & GH3 & $\begin{array}{l}\text { Transcriptional regulator (AraC family); } \\
\text { \&beta;-glucosidase (EC 3.2.1.21); glucan } \\
\text { 1,4-\&beta;-glucosidase (EC 3.2.1.74) }\end{array}$ & $\begin{array}{l}\text { Cohnella sp. CIP } \\
111063^{\text {bc }}\end{array}$ & [19] \\
\hline FP.3.3_22593 & 3.2.1.21 & GH3 & $\begin{array}{l}\text { Transcriptional regulator (AraC family); } \\
\text { \&beta;-glucosidase (EC 3.2.1.21); glucan } \\
\text { 1,4-\&beta;-glucosidase (EC 3.2.1.74) }\end{array}$ & $\begin{array}{l}\text { Cohnella sp. CIP } \\
111063^{\mathrm{bc}}\end{array}$ & [19] \\
\hline FP.3.2_23567 & 3.2.1.86 & GH4 & $\begin{array}{l}\text { 6-phospho-\&beta;-glucosidase } \\
3.2 .1 .86 \text { ) }\end{array}$ & $\begin{array}{l}\text { Cohnella sp. CIP } \\
111063^{\text {bc }}\end{array}$ & [19] \\
\hline FP.3.1_15556 & 3.2.1.74 & GH116 & glucosidase (EC 3.2.1.74); & $\begin{array}{l}\text { Cohnella sp. CIP } \\
111063^{\text {bc }}\end{array}$ & [19] \\
\hline
\end{tabular}


FP.3.3_21908

3.2.1.86

$\mathrm{GH} 4$

6-phospho-\&beta;-glucosidase

(EC Cohnella

sp.

[19]

3.2.1.86)

OV330

Top 10 genes belong to abundance disturbing pattern 14 .

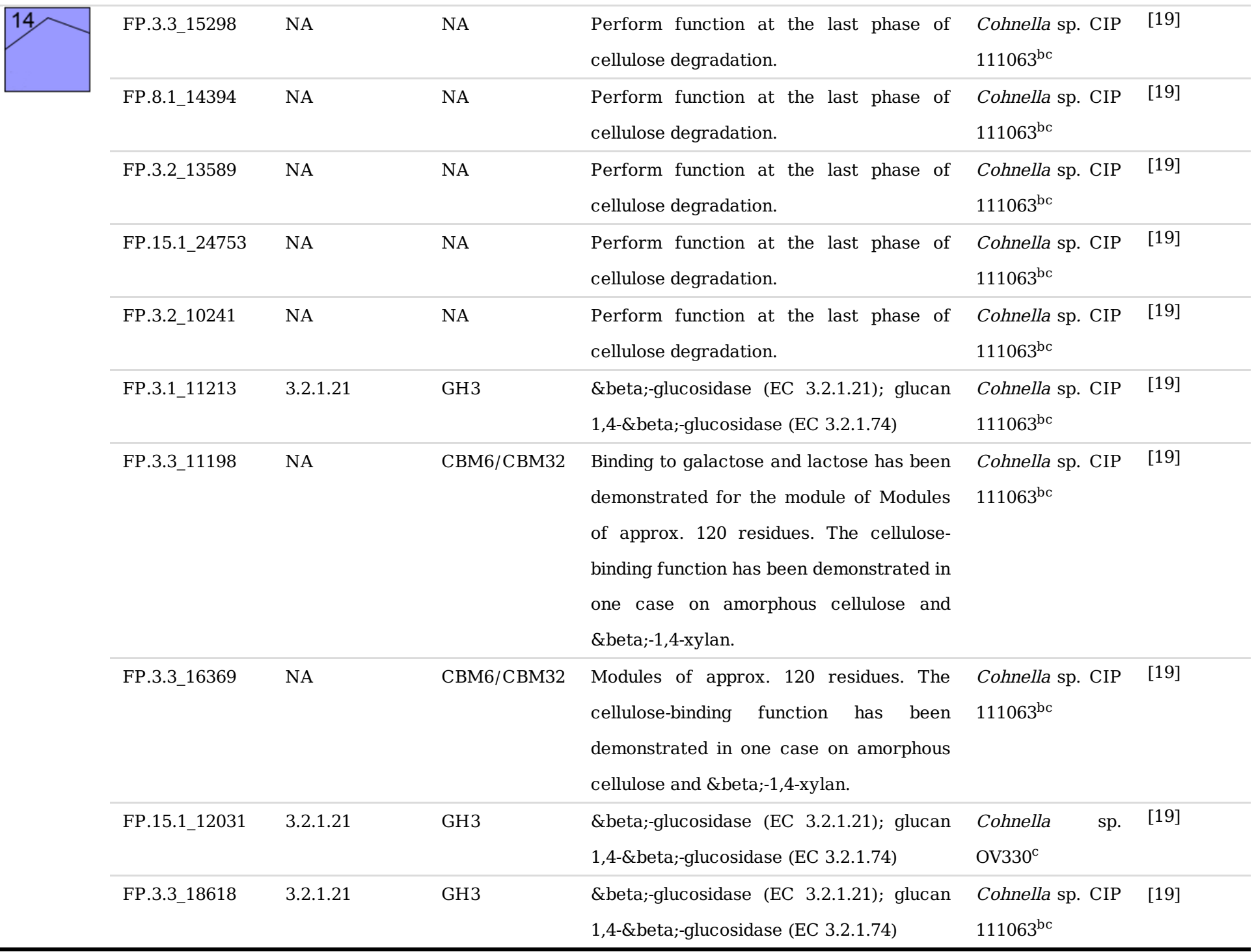

Putative function genes (PCGs) were highlighted in gray.

a was the variation pattern of gene abundance clustered by STEM algorithm, the significant patterns of $0,4,11$ and 14 were displayed, and the top 5 of relative abundance of PCGs and the top 5 of relative abundance of candidate cellulolytic genes were listed.

${ }^{\mathrm{b}}$ represents the species that carry the cellulose-related genes detected in microbiota FP, including cellulases and exoglucanases.

${ }^{\mathrm{c}}$ represents the species that carry the cellobiose-related genes detected in microbiota FP, including beta-glucosidases and phosphocellobiase.

Figures 


\section{Day 3}
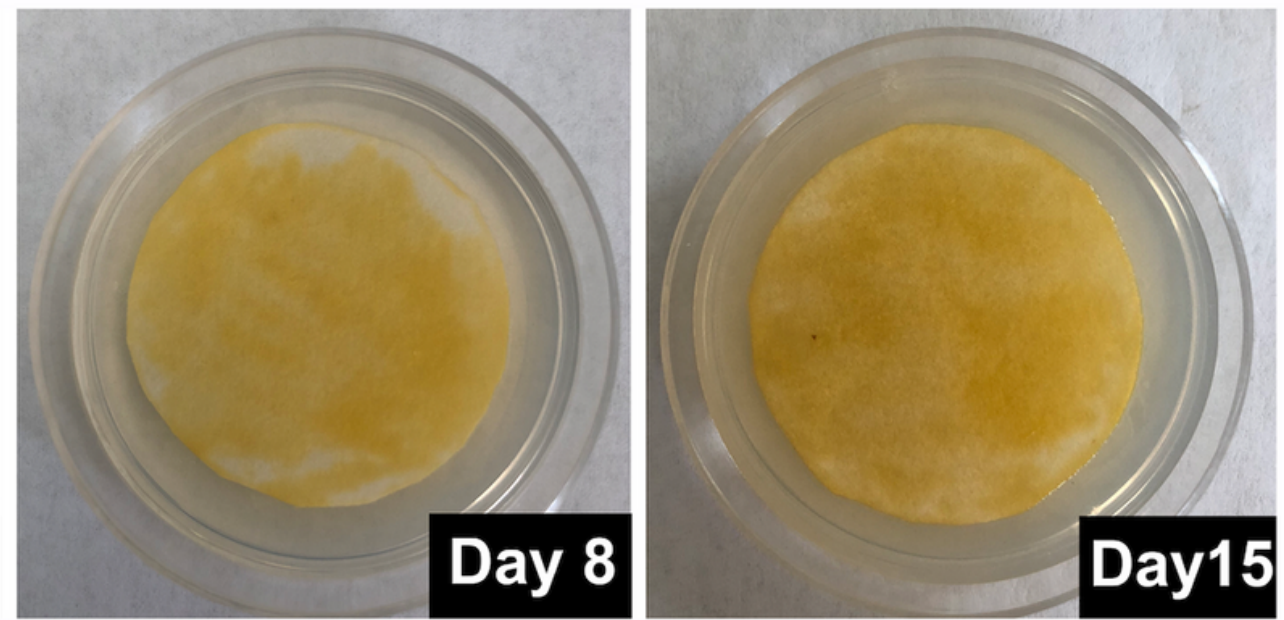

Figure 1

The degradation process of filter paper by microbiota FP. The microbiota was cultivated in filter paper medium at $30^{\circ} \mathrm{C}$ for 3,8 and 15 days.

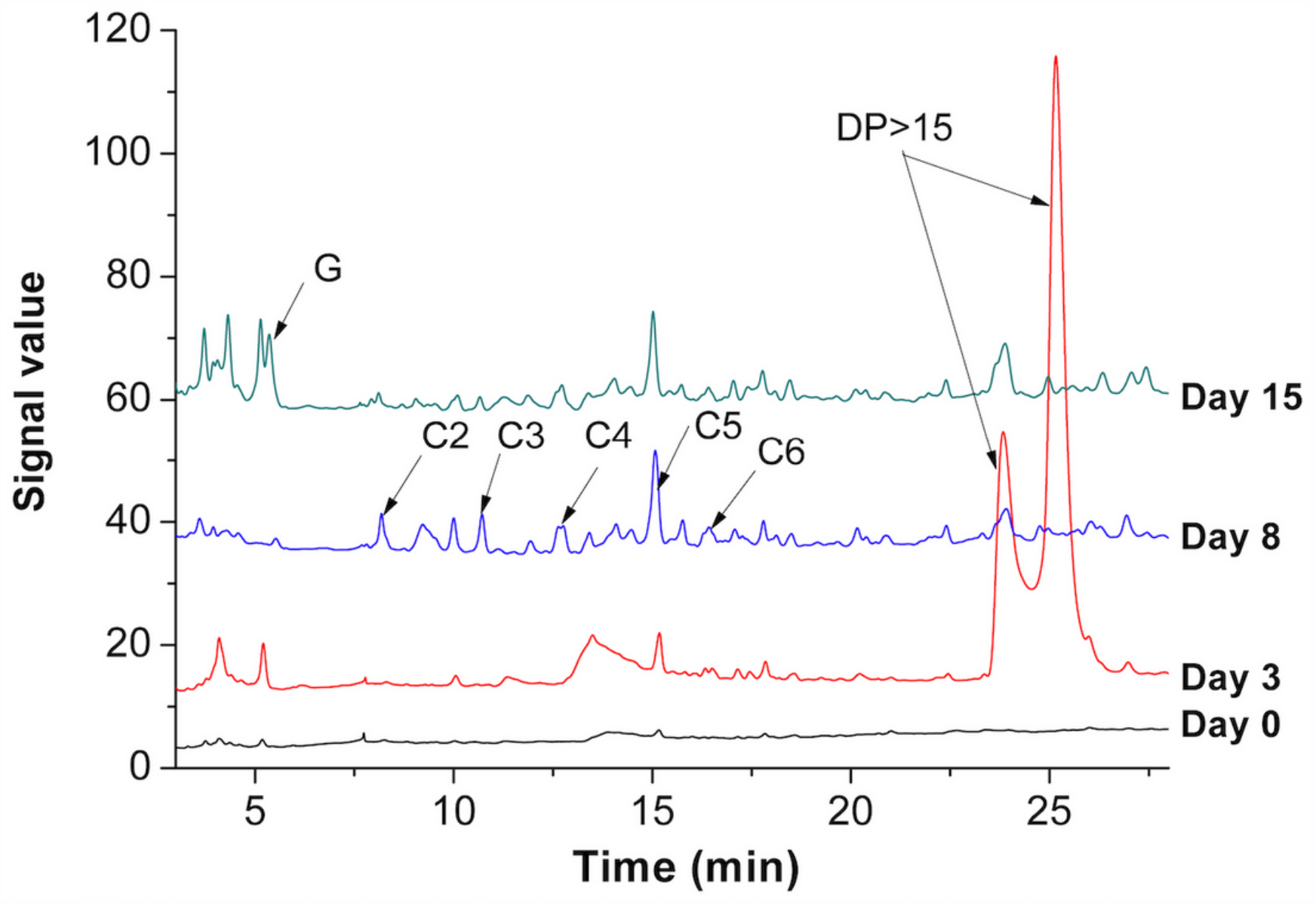

Figure 2 

$30^{\circ} \mathrm{C}$ for 3,8 , and 15 days. Abbreviations: $\mathrm{G}$ represents glucose, $\mathrm{C} 2-\mathrm{C} 6$ refers to cello-oligosaccharides with DP from 2 to 6.

a
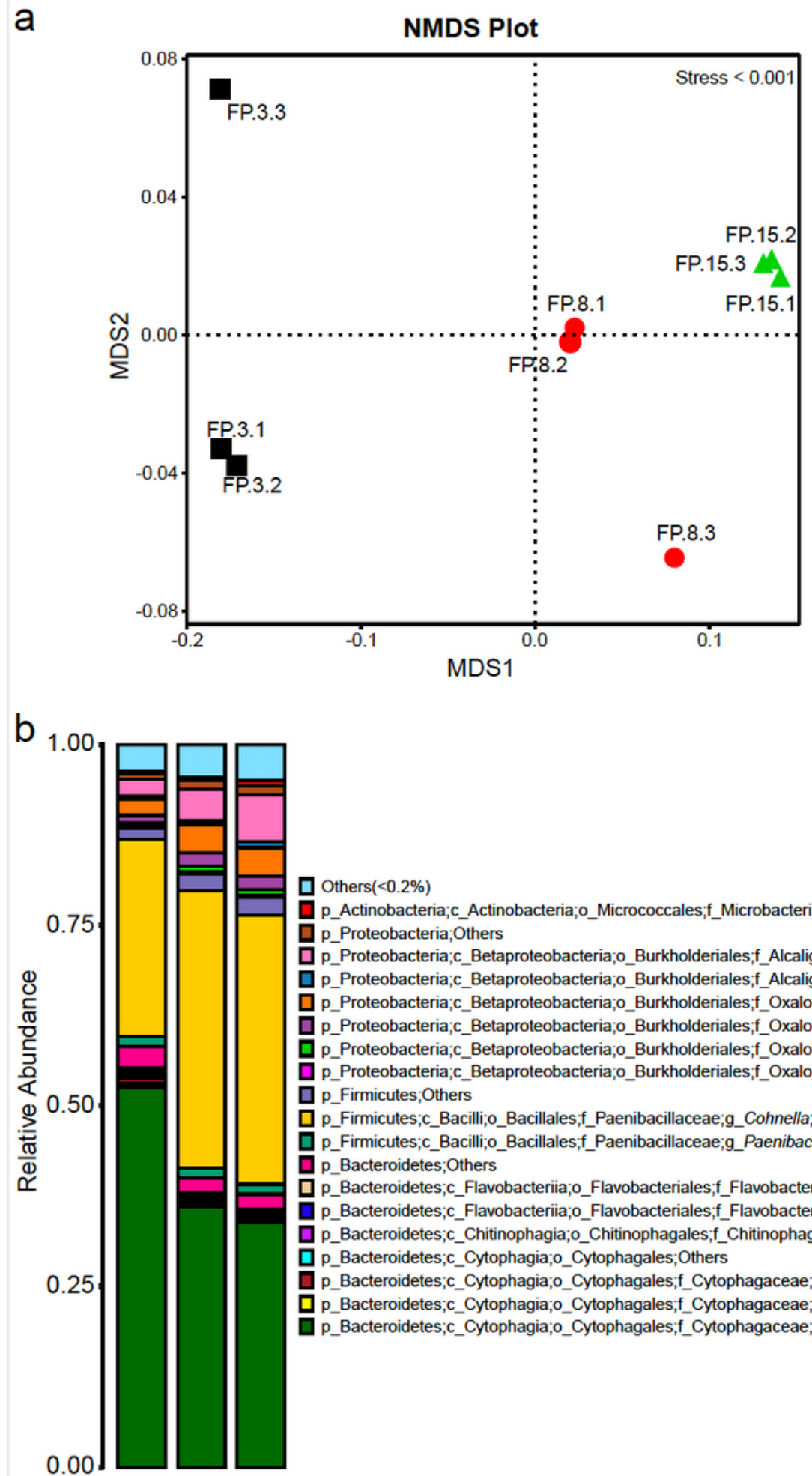

D Others $(<0.2 \%)$

p_Actinobacteria;c_Actinobacteria;o_Micrococcales,f_Microbacteriaceae;g_Microbacterium;s_M. sp. XT11

p_Proteobacteria; Others

p_Proteobacteria;c_Betaproteobacteria;o_Burkholderiales,f_Alcaligenaceae;g_Achromobacter,Others

口 p_Proteobacteria;__Betaproteobacteria;o_Burkholderiales,f_Alcaligenaceae;g_Achromobacter,s_A. denitrificans

$\square$ p_Proteobacteria;c_Betaproteobacteria;o_Burkholderiales,f_Oxalobacteraceae;g_Herbaspirillum;Others

p_Proteobacteria;c_Betaproteobacteria;o_Burkholderiales,f_Oxalobacteraceae;g_Herbaspirillum;s_H. huttiense

口 p_Proteobacteria;c_Betaproteobacteria;o_Burkholderiales,f_Oxalobacteraceae;g_Herbaspirillum;s_H. sp. B39

p_Proteobacteria;c_Betaproteobacteria;o_Burkholderiales;f_Oxalobacteraceae;g_Herbaspirillum;s_H. aquaticum

p_Firmicutes;Others

p_Firmicutes;c_Bacilli;o_Bacillales;f_Paenibacillaceae;g_Cohnella;s_C. sp. CIP 111063

p_Firmicutes;c_Bacilli;o_Bacillales;f_Paenibacillaceae;g_Paenibacillus;Others

P_Bacteroidetes; Others

p_Bacteroidetes;c_Flavobacteriia;o_Flavobacteriales,f_Flavobacteriaceae;Others

D_Bacteroidetes;c_Flavobacteriia;o_Flavobacteriales;_fFlavobacteriaceae;g_Flavobacterium;Others

P_Bacteroidetes;__Chitinophagia;o_Chitinophagales,__Chitinophagaceae;g_Chitinophaga;Others

D p_Bacteroidetes;c_Cytophagia;o_Cytophagales;Others

P_Bacteroidetes;_C_Cytophagia;__Cytophagales,f_Cytophagaceae;Others

p_Bacteroidetes;c_Cytophagia;o_Cytophagales,f_Cytophagaceae;g_Cytophaga;Others

p_Bacteroidetes;c_Cytophagia;o_Cytophagales;__Cytophagaceae;g_Sporocytophaga;s_S. myxococcoides

FP.3 FP.8 FP.15

Figure 3 
Structure analysis of microbiota FP. a) Nonmetric multidimensional scaling (NMDS) plot based on BrayCurtis dissimilarities of microbiota composition. Each point represents a single sample, and each color corresponds to a treatment. Stress values less than 0.2 indicate acceptable confidence in the observed groupings. b) Relative abundances of the dominant microbial taxa. Each bar represents the average relative abundance of a treatment. Taxa with relative abundances greater than $0.2 \%$ are shown.

Abbreviations: FP.3, FP.8 and FP.15 refer to FP microbiota cultivated in fiber medium for 3, 8, and 15 days, respectively.

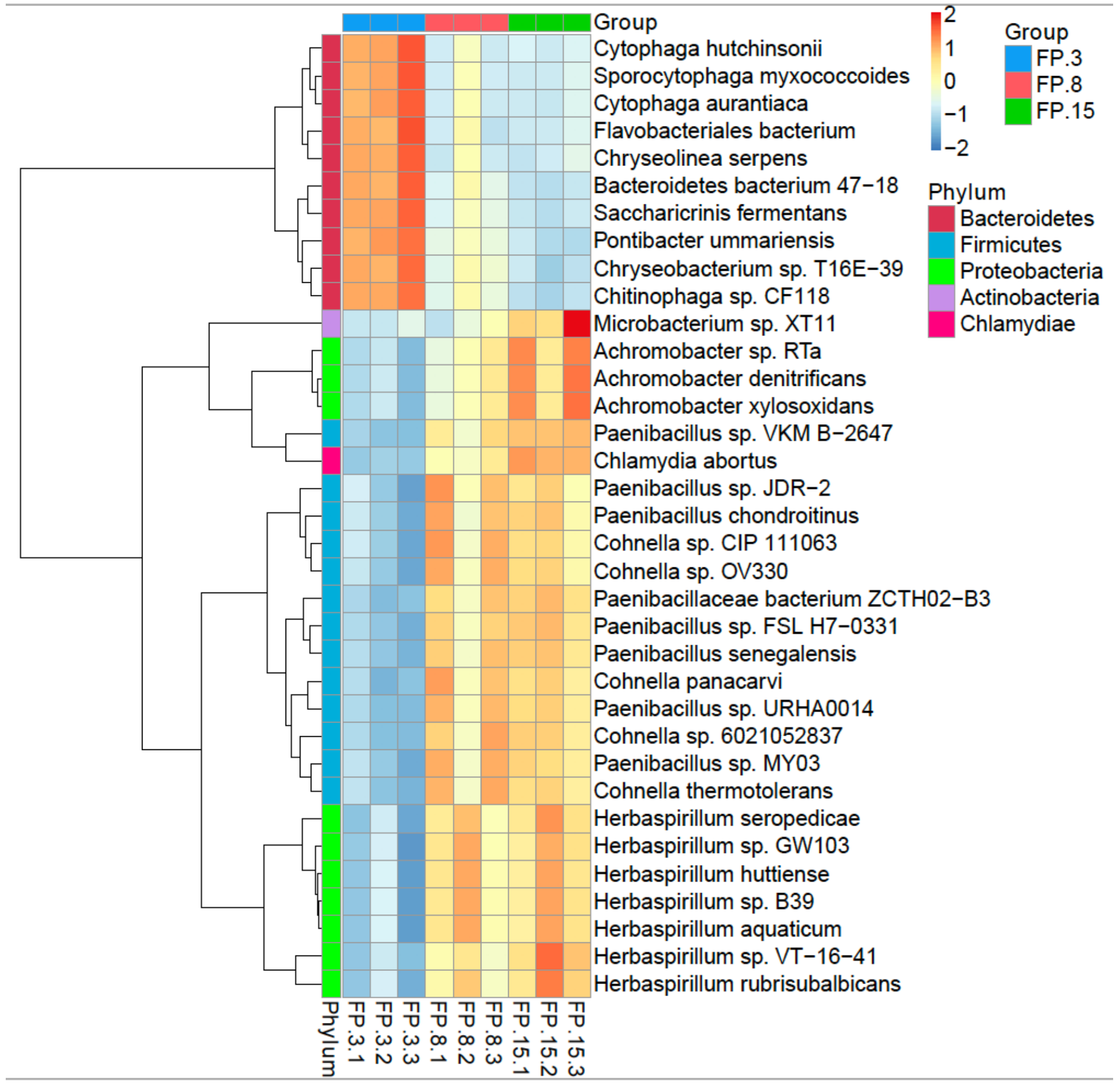

Figure 4 
Heat map of species relative abundance of microbiota FP at different cellulose-degrading stages. The tree on the left is clustered based on the change pattern of the relative abundance of species, and the color intensity (log scale) of each panel is proportional to a z-value calculated by visualizing the relative abundance of a species in the corresponding sample, with reference to the color bar (top right).

Specifically, FP.3, FP.8 and FP.15 refer to FP cultivated in filter paper medium for 3, 8, and 15 days, respectively.

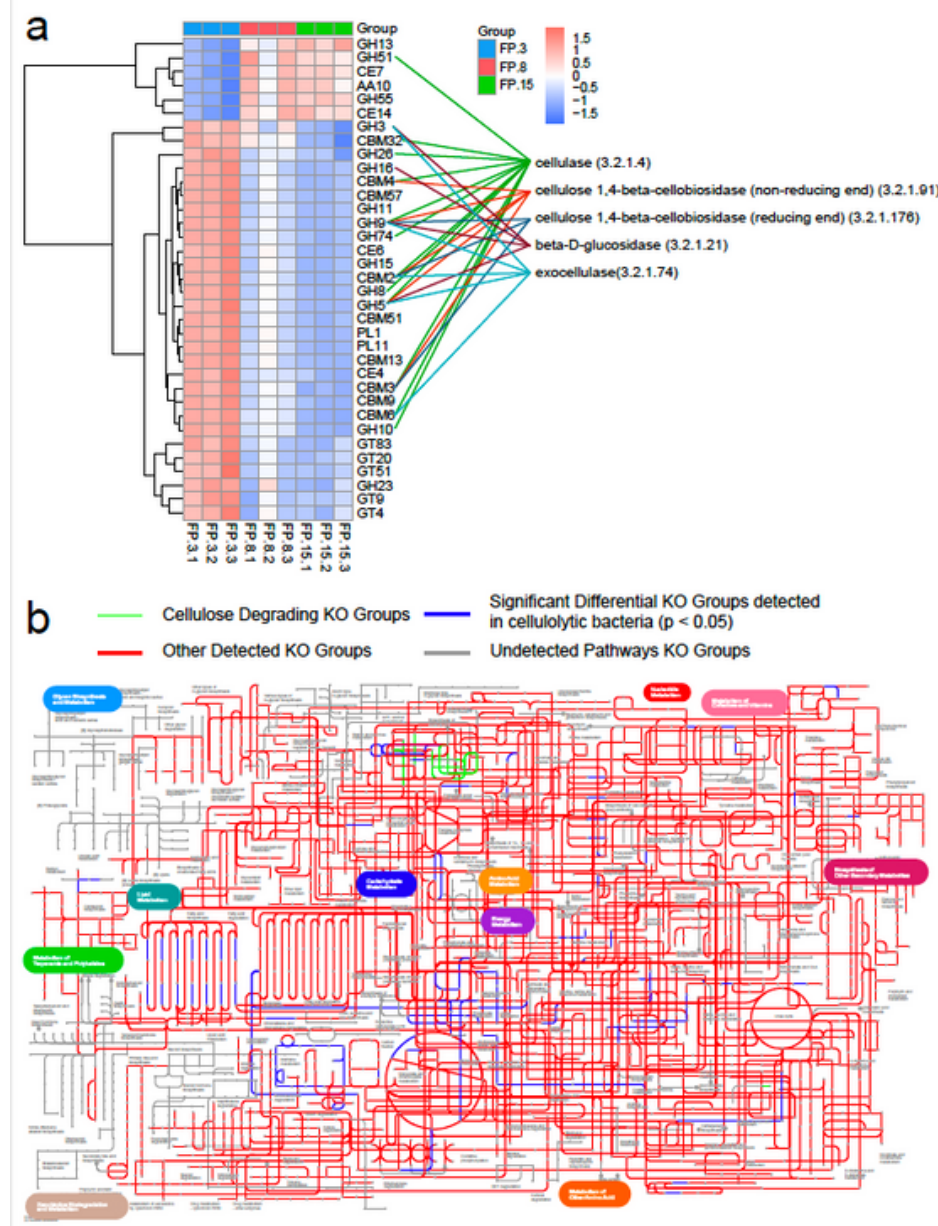

C

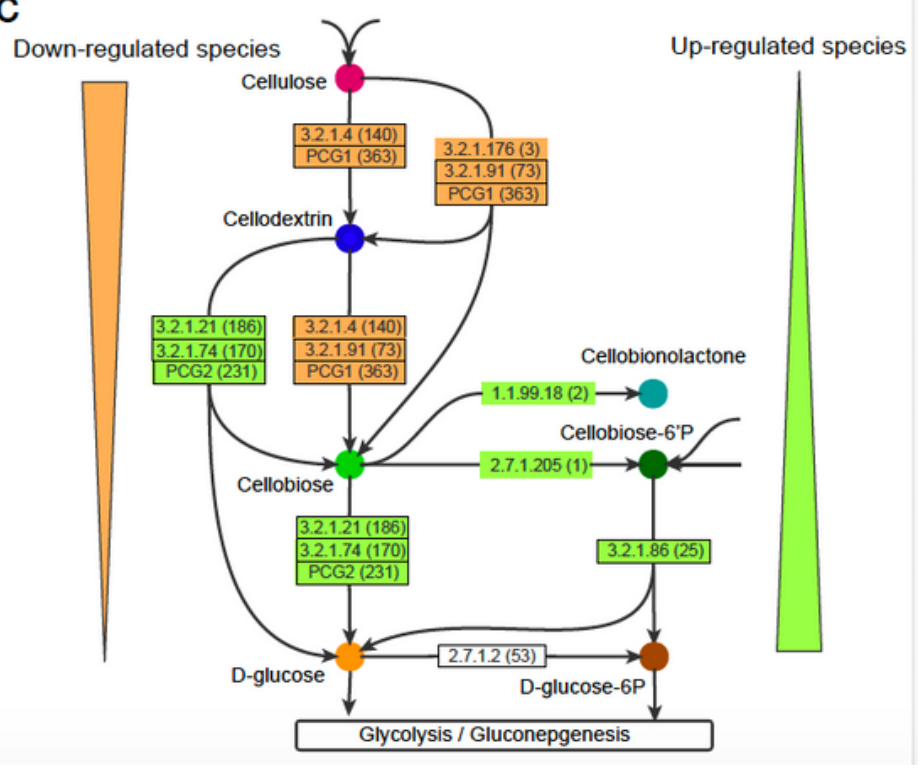

Figure 5 
The cellulolytic function of microbiota FP. a) Heat map of the relative abundance of the top 35 carbohydrate-active enzymes (CAZy) and CBM families from microbiota FP. The tree on the left is clustered based on the change pattern of the relative abundance of species. Each panel shows a z-value calculated by normalizing the relative abundance and translating it into color intensity (log scale), with reference to the color bar (top right). b) Projection of the FP metagenome on the KEGG pathways. The red and blue lines represent KEGG orthology (KO) groups with and without significant differences between treatments respectively, and the green lines represent the KO groups related to cellulose degradation. $c$ ) Cellulolytic catabolism pathway of microbiota FP. Different colored dots represent different metabolites. Arrows indicate both the pathways and directions of metabolism. The numbers in colored boxes refer to the EC number of the enzymes. Numbers in brackets represent the number of genes identified. PCG1 and PCG2 represent putative cellulolytic genes with different functions. Orange and light green wedges represent the pathways of the downregulated species (Sporocytophaga myxococcoide) and the upregulated species (Cohnella sp. CIP 111063). The width of the wedges roughly represents the species' contribution rate to the metabolic pathway parallel to the corresponding position. 